\title{
SOMALI HEALTH ACTION JOURNAL - A Collaborative Venture for Health Research and Development
}

$\mathrm{W}$ elcome to the Somali Health Action Journal (SHAJ), an open-access online publication. The SHAJ offers a national and international platform for knowledge sharing on key health system topics in the fragile and post-conflict context of Somali health development and other similar settings. It aims to pave the way for interaction between Somali academics and their institutions, health system professionals, policy makers and international partners, to develop local evidence-based solutions for the health problems of the population. The Journal's goals include building collaborative health research partnerships and a research culture that focuses on priority health needs. It also aims to promote research capacity building across academic health institutions and cross-university research collaborations.

The idea of this Journal evolved from a few international seminars and workshops, held in Sweden from 2014 and onwards. The participants included representatives from six Somali and five Swedish universities as well as Somali diaspora professionals. The aim was to revive a Somali-Swedish research cooperation programme which was terminated in the early 1990s due to civil unrest. Focusing on health and the challenges met in rebuilding the Somali health and health care system, the discussions pointed to the importance of public health oriented research and the crucial role of universities in building capacity for this purpose $(1,2)$. This led to the organization of a joint health research training programme. The first course enabled young academics from Somali universities and health services to successfully complete and report on field projects under the guidance of Somali and Swedish mentors. In the absence of a local channel for the dissemination of research findings, so far two such reports have been published in an international journal $(3,4)$, and others are in progress.

While recognizing the scarcity of research publications, particularly by Somali authors dealing with Somali health problems, the parties decided to collaborate and create a platform for the dissemination of research and policy debates. During the planning process, it was agreed that the ownership of SHAJ shall rest with Somali universities. However, for practical reasons, on an interim basis the Journal will initially be hosted by the Somali-Swedish Researchers' Association, which is a Swedish based non-governmental organization. The SHAJ initiative has also been supported by the World Health Organization's country leadership.

To start with, several commissioned articles will focus on pertinent health issues and interventions in the Somali context. These articles will include reports from on-going projects on diverse issues such as mental health, female genital mutilation/cutting, strategies for post-conflict development of the health system and malaria control in Somalia. An on-going bibliometric review will assess who has published what on health 
and health care in Somalia over the past four decades. Discussion papers will be invited on policy options for re-building the health system and the role of research networking in this process.

The need for research dissemination is a critical reason for launching the Journal and we expect to catalyze the national aspirations towards attainment of Universal Health Coverage (UHC) and other Sustainable Development Goals (SDGs) inextricably linked to health by the year 2030. The Journal will voice the opinions of many Somali health professionals and academic institutions operating for decades in their fragile health system with limited research capacity. The ensuing digital platform will also provide young Somali scholars and health care workers with access to evidence-based health information.

\section{SHAJ as a lever for health system recovery}

The Journal will serve as a forum for documentation and assessment of health care delivery and health research addressing priority issues. In the Somali context, the burden of disease approach, predominantly designed for diseases affecting the poor and underprivileged, is an important pathway. The SHAJ, in its role as a public health envoy, will promote and focus on the following:

- Research addressing the high burden of health challenges aligned with local health priorities and establishing linkages between academic institutions and stakeholders from the public and private health sectors;

- Community-based research, as a vital strategy for developing effective and culturally relevant and essential health interventions and for advancing the roles and responsibilities of health authorities in achieving UHC with the Essential Package of Health Services, and

- Studies on the district primary health care (PHC) services, generating research evidence to create effective strategies in the local contexts for integrating the vertical health programs into the PHC model.

\section{Scope and papers sought}

The SHAJ seeks manuscripts addressing national health priorities and challenges confronting the Somali health system, bringing research analyses and recommendations about its strengthening to the fore. Authors, not least locally resident researchers, are hence encouraged to submit:
- Original Articles aligned with the SDGs and UHC priority interventions reaching out to the underprivileged and hard to access communities, using quantitative, qualitative, or mixed research designs;

- Short Communications from pilot studies, fieldoriented surveys, qualitative interviews, and evidence arising out of contextual experiences adding value and knowledge relevant to implementation processes;

- Capacity Building papers documenting how essential research infrastructure and capability, as well as human resources in general, can be developed as a basis for public health work in a fragile country;

- Networking experiences reflecting on how partnerships are beneficial in a post-conflict country;

- Educational Articles that outline the design, implementation, and evaluation of initiatives to improve the competence and performance levels of the health workforce and the services they provide;

- Review Articles on health promotion, disease prevention, and the diagnosis and care of diseases of public health importance;

- Debate Articles sharing critical reflections about public health issues and policies of relevance for fragile states;

- Guideline Articles of relevance for public health and evidence-based health care, and

- Perspectives in Public Health articles presenting practice-based knowledge and opinion articles based on case studies and public health relevant news.

\section{Vision and mission}

The SHAJ will help foster public health interventions through the collaboration between key stakeholders and it will also contribute to knowledge and capacity development. The Journal aims to facilitate evidencebased implementation, placing local communities at the heart of decision making and transformation processes. The SHAJ will also encourage multisectoral health research, addressing the underlying environmental and social determinants of ill health. Our core mission is to communicate and disseminate updated evidence to influence health care decisions, policy and practice. The Journal will serve as a bridge for peace and a 
voice for reconciliation and trust-building at all levels. The participating Somali universities have expressed their commitment to the Journal's mission and to the development of a local foundation for its management.

\section{Bridging the Know-do Gap}

Knowledge translation builds on a strong partnership between the service delivery system, academic institutions and grassroot communities. Experience gained in preparing for this revived research collaboration between Somali and Swedish universities points to the possibility of collaboration on a wider, international as well as national basis (5). The current efforts to revive the public health functions of the Somali National Institute of Health are expected to improve health systems' performance and research capacity across the country. The SHAJ offers a platform to highlight research generated solutions to health problems, and to increase public awareness about the need to reduce health inequities by pursuing the strategic approach of "Health in All Policies". Together with community engagement and empowerment, this pragmatic approach will effectively bridge the know-do gap in addressing the social determinants of health.

\section{Promoting Gender Equity}

Gender mainstreaming is securing a rightful place in health research with recognition of its influence on health practices and outcomes. Somalia has a gender inequality index at 0.776 out of 1 (2012) which places the country at a highly unfavorable position (6). Research addressing Somali gender inequality issues can significantly improve our understanding of several aspects of health system design and policy. Gender mainstreaming will be promoted through SHAJ publications.

We view our Journal as an exciting new frontier for deciphering and documenting advancements in public health. The SHAJ is ready to take an active role in the struggle for a speedy recovery of the Somali health system and to help in identifying and meeting existing and emerging challenges.

For the Editorial Board,

Khalif Bile Mohamud, Editor-in-Chief

\section{References}

1. Dalmar AA, Hussein AS, Walhad SA, Ibrahim AO, Abdi AA, Ali MK et al. (2017) Rebuilding research capacity in fragile states: the case of a Somali-Swedish global health initiative, Global Health Action, 10:1, 1348693, DOI: 10.1080/16549716.2017.1348693.

2. Somali-Swedish Action Group for Health Research and Development (2015) Healing the health system after civil unrest, Global Health Action, 8:1, 27381, DOI: 10.3402/ gha.v8.27381.

3. Aden JA, Ahmed HJ, \& Östergren P-O. (2019) Causes and contributing factors of maternal mortality in Bosaso District of Somalia. A retrospective study of 30 cases using a Verbal Autopsy approach, Global Health Action, 12:1, 1672314,DOI: 10.1080/16549716.2019.1672314

4. Abdullahi MF, Stewart Williams J, Sahlèn K-G, Bile KM \& Kinsman J. Factors contributing to the uptake of childhood vaccination in Galkayo District, Puntland, Somalia (2020) Global Health Action, 13:1, 1803543, DOI: 10.1080/16549716.2020.1803543

5. Pellini A, Abdi DA, Salah G, Ali HY, Quintin KL, Hassan MA, et.al. Research in Somalia: opportunities for cooperation. SIDA Report, February 2020.

6. Launch of the Women in Global Health (WGH) Somalia Chapter: Event Report, February 2019. https://bit.ly/2Hsp1DT 